\title{
QUASI-PURE PROJECTIVE AND INJECTIVE TORSION GROUPS
}

\author{
W. P. BERLINGHOFF ${ }^{1}$ AND J. D. REID
}

\begin{abstract}
This paper characterizes quasi-pure projective (q.p.p.) and quasi-pure injective (q.p.i.) p-groups, and hence characterizes all such (abelian) torsion groups. A p-group is q.p.i. if and only if it is the direct sum of a divisible group and a torsion complete group. A nonreduced p-group is q.p.p. if and only if it is the direct sum of a divisible group and a bounded group; a reduced p-group is q.p.p. if and only if it is a direct sum of cyclic groups.
\end{abstract}

This paper is a contribution to a recent flurry of results ([1], [2], [3], [6]) concerning quasi-pure projective (q.p.p.) and quasi-pure injective (q.p.i.) abelian groups. These results, taken together, form an increasingly complete answer to a problem posed by L. Fuchs [4, v. I, Problem 17]. We recall that an abelian group $G$ is said to be quasi-pure projective if every pure exact sequence of the form

$$
0 \rightarrow P \rightarrow G \stackrel{\nu}{\rightarrow} G / P \rightarrow 0
$$

yields an exact sequence

$$
0 \rightarrow \operatorname{Hom}(G, P) \rightarrow \operatorname{Hom}(G, G) \stackrel{\nu_{*}}{\rightarrow} \operatorname{Hom}(G, G / P) \rightarrow 0 .
$$

Dually, $G$ is q.p.i. Put another way, $G$ is q.p.i. if for every pure subgroup $P$ of $G$, every map $\phi: P \rightarrow G$ is the restriction of an endomorphism of $G$.

In [2] the torsion free q.p.p. and q.p.i. groups of rank 2 were characterized, and in [1] the torsion free q.p.p. and q.p.i. groups of arbitrary finite rank were characterized. In this paper, we characterize the q.p.p. and q.p.i. torsion groups. In contrast to the case of torsion-free groups, the results here are that the only torsion q.p.p. (q.p.i.) groups are the obvious ones.

All groups under discussion are torsion abelian groups, and we use notation and standard results from [4], frequently without further reference. We would like to acknowledge that we have been told that similar results to ours have also been obtained by K. Benabdallah and R. Bradley [3] but we have not seen these results and do not know how our methods compare.

Received by the editors April 23, 1976.

AMS (MOS) subject classifications (1970). Primary 20K10, $20 \mathrm{~K} 30$.

Key words and phrases. Abelian group, pure exact sequence, quasi-projective, quasi-injective, torsion complete, direct sum of cyclic groups.

'This paper is based in part on parts of the first named author's Ph. D. dissertation, Wesleyan University, 1976. 
1. Preliminary results. It follows from [1] and [6], and will follow from our results as well, that the classes of q.p.p. and of q.p.i. groups are not closed under taking direct sums. However it is routine to verify that a summand of a group in one of these classes is again in the class, and that a torsion group belongs to one of the classes if and only if each of its primary components is in the class. Therefore we will limit our attention to $p$-groups and we collect in this section the easy cases.

THEOREM 1. The p-group $G$ is q.p.i. if and only if $G=D \oplus R$ where $D$ is divisible and $R$ is torsion complete.

Proof. Let $G$ be q.p.i. and suppose first that $G$ is reduced. If $B_{1}$ and $B_{2}$ are any two basic subgroups of $G$, and $\alpha: B_{1} \rightarrow B_{2}$ is an isomorphism, then $\alpha$ can be extended to an endomorphism, again denoted by $\alpha$, of $G$. Writing $\beta$ : $B_{2} \rightarrow B_{1}$ for the inverse of $\alpha$, we extend $\beta$ to $G$, the extension being denoted by $\beta$ as well. Then $1-\alpha \beta$ is an endomorphism of $G$ which annihilates $B_{2}$. Since $G$ is reduced, we conclude that $\alpha \beta=1$. Similarly $\beta \alpha=1$ so that $\alpha$ is an automorphism of $G$. It follows (e.g. $\$ 69$ of [4]) that $G$ is torsion complete.

Now if $G$ is any q.p.i. $p$-group and $D$ is its maximal divisible subgroup, we may write $G=D \oplus R$ with $R$ reduced and, according to our remarks above, q.p.i. Hence $R$ is torsion complete.

Conversely if $G=D \oplus R$ with $D$ divisible and $R$ torsion complete, any pure exact sequence

$$
0 \rightarrow H \rightarrow G \rightarrow G / H \rightarrow 0
$$

yields an exact sequence

$$
0 \rightarrow \operatorname{Hom}(G / H, G) \rightarrow \operatorname{Hom}(G, G) \rightarrow \operatorname{Hom}(H, G) \rightarrow \operatorname{Pext}(G / H, G)
$$

Certainly $\operatorname{Pext}(G / H, D)=0$. Since $R$ is torsion complete and $G / H$ is a $p$-group, $\operatorname{Pext}(G / H, R)=0$ too. Thus

$$
\operatorname{Pext}(G / H, G)=\operatorname{Pext}(G / H, D) \oplus \operatorname{Pext}(G / H, R)=0
$$

so $G$ is q.p.i.

THEOREM 2. A nonreduced p-group $G$ is q.p.p. if and only if $G=D \oplus B$ where $D$ is divisible and $B$ is bounded.

Proof. If $G=D \oplus B$ with $D$ divisible and $B$ bounded then every pure subgroup of $G$ has the same form, hence is a summand of $G$. It is clear from this that such groups $G$ are q.p.p.

Conversely suppose $G$ is a q.p.p. p-group and write $G=D \oplus R$ with $D$ divisible and $R$ reduced. Let $B$ be basic in $R$ and suppose $B \neq R$. Select a summand $X \approx Z\left(p^{\infty}\right)$ of $D$ and a summand $Y \approx Z\left(p^{\infty}\right)$ of $G / D \oplus B$. These are isomorphic by a map which extends to a homomorphism $\theta$ : $G \rightarrow G / D \oplus B$, since $G / D \oplus B$ is divisible. Since $G$ is q.p.p., there is an endomorphism $\alpha$ of $G$ such that $\nu \alpha=\theta$, where $\nu: G \rightarrow G / D \oplus B$ is the natural map. Then $\nu \alpha(X)=\theta(X)=Y$, whereas $\alpha(X) \subseteq D \subseteq \operatorname{ker} \nu$ so $\nu \alpha(X)$ 
$=0$. We conclude that $R$ has no proper basic subgroups and therefore, as is well known, is bounded.

2. Reduced q.p.p. $p$-groups. It remains only to consider the case of reduced q.p.p. $p$-groups, and we denote such a group by $G$ throughout this section.

LEMMA 1. G has no elements of infinite height.

Proof. Denote the subgroup of elements of infinite height in $G$ by $F$, and let $B$ be basic in $G$. Since $G$ is q.p.p. and $B$ is pure in $G$, to every endomorphism $\theta$ of $G / B$ there corresponds an endomorphism $\alpha$ of $G$ such that $\nu \alpha=\theta \nu$, where $\nu: G \rightarrow G / B$ is the natural map. Such maps $\alpha$ clearly satisfy $\alpha B \subseteq B$ and since $F$ is fully invariant in $G$, we conclude that $F+B / B=\nu(F)$ is fully invariant in $G / B$. Since $G / B$ is divisible, this implies that $\nu(F)=G / B$, or $\nu(F)=(G / B)\left[p^{n}\right]$ for some $n$.

If $\nu(F)=G / B$, then $F+B=G$ and since $F \cap B=0, F$ is a summand, hence pure, in $G$. But then $F$ is divisible so $F=0$ since $G$ is reduced. Suppose then that $\nu(F)=(G / B)\left[p^{n}\right]$. Clearly $B$ is unbounded, otherwise $G=B$ since $G$ is reduced, and we are done. Then $B$ has a basic subgroup $B^{\prime}$ distinct from itself. $B^{\prime}$ is basic in $G$ as well, so our remarks to date applied to $B^{\prime}$ yield $\left(F+B^{\prime}\right) / B^{\prime}=\left(G / B^{\prime}\right)\left[p^{m}\right]$ for some $m>0$. In particular, $G[p] \subseteq F+B^{\prime}$. It follows that $B[p] \subseteq B^{\prime}$ and the purity of $B^{\prime}$ now forces $B^{\prime}=B$, contrary to the choice of $B^{\prime}$. This proves the lemma.

The proof of our main theorem does not really require Lemma 1. Rather, what is needed is the fact that our group $G$ satisfies fin $r(G)=\operatorname{fin} r(B)$ for $B$ basic in $G$. This will follow from Lemma 2 below whose proof, however, uses the generalized continuum hypothesis. We have included Lemma 1 since it settles the issue immediately for countable groups, and even for groups $G$ such that $G / B$ is countable for some basic subgroup $B$ of $G$ (cf. $[4, \S 68])$. It may also turn out to be of use in a proof of Theorem 3 which would avoid GCH.

LEMMA 2. If $B$ is basic in $G$, then $|B|=|G|$.

Proof. Let $B$ be basic in $G$ and assume $|B|<|G|$. Put $D=G / B$ so that $|D|=|G|$. Since $G$ is q.p.p. and $B$ is pure in $G$ we have, as above, for every endomorphism $\theta$ of $D$ an endomorphism $\alpha$ of $G$ such that $\nu \alpha=\theta \nu, \nu$ the natural map of $G$ onto $D$. As remarked earlier, $\alpha \in E(G, B)=\{\sigma \in$ $E(G) \mid \sigma B \subseteq B\}$. Thus we have an isomorphism

$$
E(D) \approx E(G, B) / \operatorname{Hom}(G, B)
$$

Since $G$ is reduced, restriction gives a monomorphism of $E(G, B)$ into $E(B)$ so that $|E(G, B)| \leqslant|E(B)|$. It is well known that $|E(D)|=2^{|D|}$ and $|E(B)|$ $=2^{|B|}$ so that $2^{|D|}=|E(D)| \leqslant|E(G, B)| \leqslant|E(B)| \leqslant 2^{|B|}$. However, the inequality $2^{|D|} \leqslant 2^{|B|}$, viewed in the light of our assumption $|B|<|G|$ (hence $|D|=|G|$ ), violates $\mathrm{GCH}$. This proves the lemma. 
Proposition 1. Let $C$ be a q.p.p. group and let $R$ be pure in $C$. Then $C / R$ is q.p.p. if and only if the following condition holds:

... if $L$ is a pure subgroup of $C$ containing $R$, and $\varphi$ is an endomorphism of $C$ with $\varphi(R) \subseteq L$, then there exists an endomorphism $\sigma$ of $C$ such that $\sigma(R) \subseteq R$ and $(\varphi-\sigma)(C) \subseteq L$.

The proof of this proposition is routine, and we omit it. The condition (*) is simply a translation of the fact that $C / R$ is q.p.p. to a statement about endomorphisms of $C$.

Our next lemma is a corollary to a theorem of Hill ([5]; see also [7]). Hill's theorem states that if $C$ is a direct sum of cyclic groups, $B_{i}$ are basic in $C$ $(i=1,2)$ and $C / B_{1} \approx C / B_{2}$ then there is an automorphism $\gamma$ of $C$ such that $\gamma\left(B_{1}\right)=B_{2}$. We need this result in the following generalized form.

LEMMA 3. Let $C$ be a direct sum of cyclic groups with $L$ basic in $C$. Let $B_{1}$ and $B_{2}$ be basic in $L$, such that $C / B_{1} \approx L / B_{2}$. Then there exists an isomorphism $\varphi: C \approx L$ such that $\varphi\left(B_{1}\right)=B_{2}$.

Proof. Since $C$ is a direct sum of cyclic groups and $L$ is basic in $C$, there is an isomorphism $\psi: C \approx L$. Put $B_{1}^{\prime}=\psi\left(B_{1}\right)$. Then $L / B_{2} \approx C / B_{1} \approx L / B_{1}^{\prime}$, so by Hill's theorem, there is an automorphism $\lambda$ of $L$ such that $\lambda\left(B_{1}^{\prime}\right)=B_{2}$. Then $\varphi=\lambda \psi$ satisfies our requirement.

Finally, we may normalize $G$ and assume of it that, if $B$ is basic in $G$, then $r(G)=$ fin $r(G)=r(B)=$ fin $r(B)$. To effect this, we write $G=G^{\prime} \oplus G^{\prime \prime}$ where $G^{\prime}$ is bounded and $r\left(G^{\prime \prime}\right)=$ fin $r\left(G^{\prime \prime}\right)$ (cf. [4, §35]). Then $G^{\prime \prime}$ is q.p.p. and $G$ is a direct sum of cyclic groups if $G^{\prime \prime}$ is. Thus we may assume $r(G)=$ fin $r(G)$ to begin with. Similarly, for $B$ basic in $G$, write $B=B^{\prime} \oplus$ $B^{\prime \prime}$ with $B^{\prime}$ bounded and $r\left(B^{\prime \prime}\right)=$ fin $r\left(B^{\prime \prime}\right)$. Then $G=B^{\prime} \oplus H$ for some $H$ and we have $r(G) \geqslant r(H) \geqslant$ fin $r(H)=$ fin $r(G)=r(G)$ so $r(H)=$ fin $r(H)$. Since $B^{\prime \prime}$ is isomorphic to a basic subgroup of $H$, we have replaced $G$ by a q.p.p. group $H$ satisfying $r(H)=$ fin $r(H)$ and $r(B)=$ fin $r(B)$ for $B$ basic in $H$. Moreover $G$ is a direct sum of cyclic groups if and only if $H$ is. By Lemma $2,|B|=|H|$, and hence $r(B)=|B|=|H|=r(H)$, so our reduction is accomplished. We now return to our original notation, denoting the q.p.p. $p$-group under discussion by $G$.

THEOREM 3. A reduced p-group $G$ is q.p.p. if and only if $G$ is a direct sum of cyclic groups.

Proof. Direct sums of cyclic groups are pure-projective, hence certainly are q.p.p. Let $G$ then be a reduced q.p.p. $p$-group. From above we may assume fin $r(G)=$ fin $r(B)$ for every basic subgroup $B$ of $G$. Taking $B$ to be a lower basic subgroup of $G$, and $B^{\prime}$ to be a lower basic subgroup of $B$, we therefore have $G / B \approx B / B^{\prime}$ since $r(G / B)=$ fin $r(G)$, and fin $r(B)=r\left(B / B^{\prime}\right)$.

Now let

$$
0 \rightarrow R \rightarrow C \stackrel{\eta}{\rightarrow} G \rightarrow 0
$$


be a pure exact sequence with $C$ a direct sum of cyclic groups (cf. [4, §30]). Since $|G|=\operatorname{fin} r(G), G$ has a pair $B_{1}, B_{2}$ of disjoint basic subgroups (cf. [4, §35]), which we may take to be lower. Applying the remark above to these, we choose $B_{i}^{\prime}$ basic in $B_{i}$ such that $G / B_{i} \approx B_{i} / B_{i}^{\prime}(i=1,2)$. Let $L_{i}=$ $\eta^{-1}\left(B_{i}\right), L_{i}^{\prime}=\eta^{-1}\left(B_{i}^{\prime}\right)$.

Then $L_{i}$ is basic in $C, L_{i}^{\prime}$ basic in $L_{i}$ and $C / L_{i} \approx L_{i} / L_{i}^{\prime}, i=1,2$. By Lemma 3 there are isomorphisms $\varphi_{i}: C \approx L_{i}$ such that $\varphi_{i}\left(L_{i}\right)=L_{i}^{\prime}, i=1,2$. Since $R \subset L_{i}^{\prime}$, we may apply Proposition 1 to write $\varphi_{i}=\sigma_{i}+\rho_{i}$, where $\sigma_{i}, \rho_{i} \in E(C)$ and $\sigma_{i}(R) \subseteq R, \rho_{i}(C) \subseteq L_{i}^{\prime}$.

Clearly the map $\theta_{i}$ induced in $G$ by $\sigma_{i}$ has image contained in $B_{i}$. The kernel of $\theta_{i}$ is the image under $\eta$ of $\left\{x \in C \mid \sigma_{i}(x) \in R\right\}$. If $\sigma_{i}(x) \in R$ then $\varphi_{i}(x)=$ $\sigma_{i}(x)+\rho_{i}(x) \in R+L_{i}^{\prime} \subseteq L_{i}^{\prime}=\varphi_{i}\left(L_{i}\right)$, and since $\varphi_{i}$ is monic, we have $x \in$ $L_{i}$. Thus the kernel of $\theta_{i}$ is contained in $B_{i}$.

To summarize, we now have two endomorphisms $\theta_{1}, \theta_{2}$ of $G$ into the disjoint basic subgroups $B_{1}, B_{2}$ with $\operatorname{ker} \theta_{i} \subseteq B_{i}$. These induce $\theta_{1} \times \theta_{2}: G \rightarrow$ $B_{1} \times B_{2}$ with kernel contained in $B_{1} \cap B_{2}=0$. Thus $G$ is isomorphic to a subgroup of the direct sum of cyclic groups $B_{1} \oplus B_{2} \approx B_{1} \times B_{2}$, so $G$ is itself a direct sum of cyclic groups.

\section{REFERENCES}

1. D. Arnold, B. O'Brien and J. D. Reid, Torsion free abelian q.p.i. and q.p.p. groups (to appear).

2. D. Arnold, C. I. Vinsonhaler and W. J. Wickless, Quasi-pure projective and injective torsion free abelian groups of rank 2, Rocky Mountain J. Math. 6 (1976), 61-70.

3. K. Benabdallah and R. Bradley, oral communication.

4. L. Fuchs, Infinite abelian groups, Vols. I, II, Academic Press, New York, 1970.

5. Paul Hill, The covering theorem for upper basic subgroups, Michigan Math. J. 18 (1971), 187-192.

6. J. D. Reid, C. I. Vinsonhaler and W. J. Wickless, Quasi-pure projectivity and two generalizations (to appear).

7. Dalton Tarwater and Elbert Walker, Decompositions of direct sums of cyclic p-groups, Rocky Mountain J. Math. 2 (1972), 275-282.

Department of Mathematics, Southern Connecticut State College, New Haven, CONNECTICUT 06515

Department of Mathematics, Wesleyan University, Middletown, Connecticut 06457 